Pacific Journal of Mathematics

LOCALLY CIRCULAR MINIMAL SETS 


\section{LOCALLY CIRCULAR MINIMAL SETS}

\section{NeLsoN G. MARKLEY}

This paper is devoted to an analysis of the class of minimal sets which are constructed in the following way: Start with a homeomorphism of the circle without periodic points. It contains a unique minimal set. Take a finite number of copies of this minimal set. Define a homeomorphism of this space onto itself by using the original homeomorphism and a continuous rule to determine in which copy the image lies. Finally some of the pairs of doubly asymptotic orbits may be identified. In other words, minimal skew products of a specific kind of minimal cascade and a finite permutation group with some trivial identification are studied.

Their structure group is always a direct product of a circle with a finite cyclic group, and they can be characterized in terms of their dynamical properties. If no pairs of doubly asymptotic points are identified, there is a classification theorem based on four invariants. One of these invariants is an equivalence class of weakly cohomologous functions. This weakening of the usual cohomology relation gives an effective test for isomorphism for this class of minimal sets. Moreover, the minimal cohomology classes contain canonical representatives.

1. Finite skew products. A cascade is a topological space together with a homeomorphism of it onto itself. At times we will use the more general concept of a transformation group, but the phase space will always be compact and metric. Since for a cascade we want to write the homeomorphism on the left, we will write all our actions on the left. We will assume that the reader is familiar with the proximal relation, the equicontinuous structure relation, the enveloping semigroup, and the surrounding ideas. Finite sets will be important and we will denote their cardinality by $|A|$.

Let $(X, T)$ and $(Y, H)$ be transformation groups and let $F: X \times$ $T \rightarrow H$ be a continuous mapping satisfying the condition

$$
F(x, s t)=F(t x, s) F(x, t)
$$

for all $x$ in $X$ and for all $\mathrm{s}, \mathrm{t} \in T$. Then we can define a new transformation group $(X \times Y, T)$ by

$$
t(x, y)=(t x, F(x, t) y) \text {. }
$$

This transformation group will be called the skew product of $(X, T)$ and $(Y, H)$ with respect to $F$. Bronstein [3] has established the 
following:

THEOREM 1.1. Let $(X, T)$ and $(Y, H)$ be minimal transformation groups and let $F$ be as above. If the proximal relation of $(Y, H)$ is a closed equivalence relation and if $y$ is a distal point of $(Y, H)$, then $(x, y)$ is an almost periodic point of the skew product $(X \times Y, T)$.

Let $(X, \phi)$ be a minimal cascade, let $Y=\{1, \cdots, n\}$ with the descrete topology, and let $H=S_{n}$ be the permutation group on $n$ symbols. Then $\left(Y, S_{n}\right)$ is a minimal equicontinuous transformation group. A skew product of $(X, \phi)$ and $\left(Y, S_{n}\right)$ will be called a finite skew product of $(X, \phi)$. It follows from Theorem 1.1 that finite skew products are pointwise almost periodic. For finite skew products with $T=Z$, the group of integers, the defining equation takes the form

$$
F(x, m+n)=F\left(\phi^{n}(x), m\right) F(x, n) .
$$

For convenience we will denote $X \times Y=X \times\{1, \cdots, n\}$ by $X_{n}$. The finite skew of $(X, \phi)$ and $\left(Y, S_{n}\right)$ with respect to $F$ is also a cascade and its generating homeomorphism $\phi_{F}$ is given by

$$
\phi_{F}(x, i)=(\phi(x), F(x, 1) i) \text {. }
$$

Note that $F(x, 1)$ is simply a continuous function from $X$ into $S_{n}$. Conversely, let $F: X \rightarrow S_{n}$ continuously and extend $F$ to $X \times Z$ by the formulas

$$
\begin{aligned}
& F(x, 1)=F(x) \\
& F(x, 0)=e\left(e \text { denotes the identity of } S_{n}\right) \\
& F(x, n)=F\left(\phi^{n-1}(x)\right) F\left(\phi^{n-2}(x)\right) \cdots \cdots F(\phi(x)) F(x) \text { for } n>1 \\
& F(x, n)=\left[F\left(\phi^{n}(x),-n\right)\right]^{-1} \text { for } n<0 .
\end{aligned}
$$

Lemma 1.2. Let $F: X \rightarrow S_{n}$ continuously be extended to $X \times Z$ by the above formulas. Then the extension satisfies equation (1) and $\phi_{F}(x, i)=(\phi(x), F(x) i)$ is the generating homeomorphism of a finite skew product.

Proof. Equation (1) is established by considering the various cases which arise from the possible signs. If $m$ and $n$ are both nonnegative, just use the first three formulas. If they are both negative, use the fourth formula to reduce it to the nonnegative case. For the two cases of mixed signs observe that for $n<0$

$$
F(x, n)=F\left(\phi^{n}(x)\right)^{-1} F\left(\phi^{n+1}(x)\right)^{-1} \cdot \cdots \cdot F\left(\phi^{-1}(x)\right)^{-1}
$$

and then compute. 
Henceforth, $F$ will be a continuous function from $X$ into $S_{n}$, and whenever we need the above extension to $X \times \boldsymbol{Z}$ we will simply write $F(x, n)$. The skew product of $(X, \phi)$ and $\left(Y, S_{n}\right)$ with respect to $F$ will be denoted by $\left(X_{n}, \phi_{F}\right)$. Note that $\pi(x, i)=x$ is a homomorphism of $\left(X_{n}, \phi_{F}\right)$ onto $(X, \phi)$.

LemMa 1.3. If $(x, i)$ and $(y, j)$ are distinct proximal points of $\left(X_{n}, \phi_{F}\right)$, then $x$ and $y$ are proximal in $(X, \phi)$ and $x \neq y$.

\section{Proof. Obvious.}

We will say $(Y, \psi)$ is a finite extension of $(X, \phi)$ if there exists an open homomorphism $\theta$ of $(Y, \psi)$ onto $(X, \phi)$ such that $\left|\theta^{-1}(x)\right|$ is a finite constant independent of $x$. Note that a finite skew product of $(X, \phi)$ is always a finite extension of $(X, \phi)$.

THeorem 1.4. Let $(X, \phi)$ be a minimal cascade on a totally disconnected space, and let $(Y, \psi)$ be a given cascade. Then $(Y, \psi)$ is a finite extension of $(X, \phi)$ if and only if $(X, \psi)$ is a finite skew product of $(X, \phi)$.

Proof. Let $\theta$ be an open $n$ to one homomorphism of $(Y, \psi)$ onto $(X, \phi)$. It suffices to find a homeomorphism $\gamma$ of $Y$ onto $X_{n}$ such that $\gamma \circ \psi \circ \gamma^{-1}(x, i)=(\phi(x), F(x) i)$ where $F(x) \in S_{n}$. The continuity of $\gamma \circ \psi \circ \gamma^{-1}$ will guarantee the continuity of $F$. Let $\left\{y_{1}, \cdots, y_{n}\right\}=\theta^{-1}\left(x_{0}\right)$, let $U_{1}, \cdots, U_{n}$ be disjoint open neighborhoods of the $y_{i}^{\prime}$ 's, let $V$ be any open and closed subset of $X$ which is contained in $\theta\left(U_{1}\right) \cap \theta\left(U_{2}\right) \cap \cdots \cap$ $\theta\left(U_{n}\right)$, and let $W_{i}=\theta^{-1}(V) \cap U_{i}$. Then each $W_{i}$ is open and closed in $Y$ and $\theta$ maps each $W_{i}$ homeomorphically onto $V$. Cover $X$ by a finite number of open closed and disjoint subsets constructed as above, call them $V_{1}, \cdots, V_{m}$ and let $W_{1 j}, \cdots, W_{n j}$ denote the decomposition of $\theta^{-1}\left(V_{j}\right)$ into disjoint open and closed subsets of $Y$ which $\theta$ maps homeomorphically onto $V_{j}$. Set $\gamma(y)=(\theta(y), i)$ provided $y \in W_{i j}$. Then $\gamma$ is the desired homeomorphism.

CoRollaRY 1.5. Let $\left(X_{n}, \phi_{F}\right)$ be a finite skew product of the minimal cascade $(X, \phi)$ where $X$ is totally disconnected. Then every minimal set of $\left(X_{n}, \phi_{F}\right)$ is also a finite skew product of $(X, \phi)$.

2. Finite skew products of circular cascades. Let $\boldsymbol{K}$ denote the unit circle in the complex plane and let $\phi$ be a homeomorphism of $\boldsymbol{K}$ onto $\boldsymbol{K}$. If $\phi$ has no periodic points, then $(\boldsymbol{K}, \phi)$ has exactly one minimal set which is either all of $\boldsymbol{K}$ or a Cantor subset $C . \quad K \backslash C=$ $\mathbf{U}_{n=1}^{\infty}\left(a_{i}, b_{i}\right)$ where $\left(a_{i}, b_{i}\right)$ is a counterclockwise open interval in $\boldsymbol{K}$ and 
$\left[a_{i}, b_{i}\right] \cap\left[a_{j}, b_{j}\right]=\varnothing$ when $i \neq j$. The intervals $\left(a_{i}, b_{i}\right)$ are called complementary intervals of $C$. We will call all minimal sets coming from homeomorphisms of $\boldsymbol{K}$ without periodic points circular cascades, and we will use the terms transitive and intransitive to distinguish the two types. If $(K, \phi)$ is a transitive circular cascade, then $(K, \phi)$ is isomorphic to $\left(K, M_{g}\right)$ when $M_{g}(z)=g z$ and $g^{n} \neq 1$ for $n \neq 0$. If $(C, \phi \mid C)$ is an intransitive circular cascade, then two distinct points are proximal if and only if they are end points of the same complementary interval, the proximal relation equals the equicontinuous structure relation, and the maximal equicontinuous factor is of the form $\left(K, M_{g}\right)$ where $g^{n} \neq 1$ for $n \neq 0$. If we choose $g$ with $\operatorname{Im} g>0$, then it is uniquely determined for any circular cascade and will be denoted by $r(\phi)$. So for any circular cascade there exists a homomorphism $p$ of it onto $\left(K, M_{r(\phi)}\right)$ and $p(x)=p(y)$ if and only if $x$ and $y$ are proximal. Let $T(\phi)$ equal the image of the distal points under $p$. The set $T(\phi)$ is uniquely determined up to congruence. (Two sets $A$ and $B$ of $K$ are congruent, written $A \equiv B$, if there exists a $z$ such that $M_{z}(A)=B$.) Clearly $M_{r(\phi)}[T(\phi)]=T(\phi)$ and $K \backslash T(\phi)$ is countable. The author [5] has shown that $r(\phi)$ and $T(\phi)$ form a complete set of invariants for circular cascades.

Now let $(C, \phi)$ be a fixed intransitive circular cascade and let $F$ map $C$ continuously into $S_{n}$. We can find complementary intervals $\left(a_{1}, b_{1}\right), \cdots,\left(a_{p}, b_{p}\right)$ of $C$ arranged in counterclockwise order on $\boldsymbol{K}$ such that $F$ is constant on $\left[b_{1}, a_{2}\right] \cap C,\left[b_{2}, a_{3}\right] \cap C, \cdots,\left[b_{p}, a_{1}\right] \cap C$ and $F\left(a_{i}\right) \neq$ $F\left(b_{i}\right) i=1, \cdots, p$. In view of Corollary 1.5 and the pointwise almost periodicity $\left(C_{n}, \phi_{F}\right)$, we will assume $\left(C_{n}, \phi_{F}\right)$ in minimal. In this section we will be concerned with the proximal and the equicontinuous structure relations of $\left(C_{n}, \phi_{F}\right)$.

It is clear from Lemma 1.3 that $(x, i)$ is a distal point of $\left(C_{n}, \phi_{F}\right)$ if $x$ is not the end point of any complementary interval of $C$ and $\left(C_{n}, \phi_{F}\right)$ is point distal. For the end point of a complementary interval of $C$, the proximal cell is given by the following lemma.

Lemma 2.1. Let $(a, b)$ be a complementary interval of $C$. Then the following statements hold:

(a) There exists $A_{+}(a, b)\left\{A_{-}(a, b)\right\}$ in $S_{n}$ such that $\left(b, A_{+}(a, b) i\right)$ $\left\{\left(b, A_{-}(a, b) i\right)\right\}$ is the unique point of $C_{n}$ which is positively \{negatively\} asymptotic to $(a, i)$.

(b) They are the only points proximal to $(a, i)$ and they need not be distinct.

(c) For any integer $n$

$$
\begin{gathered}
A_{+}\left(\phi^{n}(a), \phi^{n}(b)\right)=F(b, n) A_{+}(a, b) F(a, n)^{-1} \\
\left\{A_{-}\left(\phi^{n}(a), \phi^{n}(b)\right)=F(b, n) A_{-}(a, b) F(a, n)^{-1}\right\} .
\end{gathered}
$$


Proof. (a) Let $P(a)$ be the smallest nonnegative integer such that $n \geqq P(a)$ implies $\phi^{n}(a) \neq a_{i} i=1, \cdots, p$. Clearly $P(a)=P(b)$. It follows that

$$
F\left(\dot{\phi}^{P(a)}(a), n\right)=F\left(\phi^{P(b)}(b), n\right)
$$

for all $n \geqq 0$. Let

$$
A_{+}(a, b)=F(b, P(b))^{-1} F(a, P(a)) .
$$

Then for $n \geqq P(a)$ we have the second coordinates of $\phi_{F}^{n}(a, i)$ and $\phi_{F}^{n}\left(b, A_{+}(a, b) i\right)$ equal which implies positive asymptoticity. Similarly one obtains $A_{-}(a, b)$.

(b) This follows from Corollary 1.5.

(c) First observe that $P\left(\phi^{n}(a)\right)=P(a)-n$ and then apply equation (1).

Let $\boldsymbol{Z}_{q}$ denote the integers modulo $q$; i.e., $\boldsymbol{Z} / q \boldsymbol{Z}$.

LEMMA 2.2. If $\psi$ is an automorphism of the topological group $\boldsymbol{K} \times \boldsymbol{Z}_{q}$, then

$$
\psi(z, i)=\left\{\begin{array}{l}
\left(\xi^{i} z, k i(\bmod q)\right) \\
\left(\xi^{i} \bar{z}, k i(\bmod q)\right)
\end{array}\right.
$$

where $\xi$ is a qth root of 1 and $k$ and $q$ are relatively prime.

THEOREM 2.3. Let $\left(\boldsymbol{K}_{q}, \psi\right)$ be a discrete flow on $\boldsymbol{K}_{q}=K \times\{1, \cdots, q\}$. If $\left(\boldsymbol{K}_{q}, \psi\right)$ has a dense orbit, then $\left(\boldsymbol{K}_{q}, \psi\right)$ is isomorphic to a unique minimal set of the form $\left(\boldsymbol{K} \times \boldsymbol{Z}_{q}, M_{\delta}\right)$ where $\delta=(g, 1), g^{n} \neq 1$ for $n \neq$ 0 , and $g=\exp (\theta i)$ with $\theta \in(0, \pi / q)$.

Proof. Clearly $\psi(\boldsymbol{K} \times\{i\})=\boldsymbol{K} \times\{\rho i\}$ where $\rho \in S_{q}$; and if $\rho$ is not a $q$-cycle, there is no dense orbit. Thus $\psi^{k}(\boldsymbol{K} \times\{1\})=\boldsymbol{K} \times\{1\}$ if and only if $q$ divides $k$ and $\left(\boldsymbol{K} \times\{1\}, \psi^{q}\right)$ has a dense orbit. Therefore, it is equicontinuous and so $\left(\boldsymbol{K}_{q}, \psi\right)$. We now know that $\boldsymbol{K}_{q}$ can be given the structure of a monothetic group and there is generator $\delta$ such that $\left(\boldsymbol{K}_{q}, \psi\right)$ is isomorphic to $\left(\boldsymbol{K}_{q}, M_{\delta}\right)$.

We can assume that $\boldsymbol{K}_{0}=\boldsymbol{K} \times\{1\}$ is the identity component, and that the group structure on $\boldsymbol{K}_{0}$ is isomorphic to complex multiplication on $\boldsymbol{K}$ [2, Theorem 2, page 19]. The cosets of $\boldsymbol{K}_{0}$ in $\boldsymbol{K}_{q}$ are $\boldsymbol{K}_{0}, \boldsymbol{K}_{0} \delta$, $\boldsymbol{K}_{0} \delta^{2}, \cdots, K_{0} \delta^{q-1}$ because $\delta^{k} \in \boldsymbol{K}_{0}$ if and only if $q$ divides $k$. There exists $h$ in $K_{0}$ such that $h^{q}=\delta^{q}$. It follows that $h^{-1} \delta$ is an element of $\boldsymbol{K}_{q}$ of order $q$ and $\boldsymbol{K}_{q}$ is isomorphic to $\boldsymbol{K} \times \boldsymbol{Z}_{q}$. Therefore, $(\boldsymbol{K}, \psi)$ is isomorphic to $\left(\boldsymbol{K} \times \boldsymbol{Z}_{q}, M_{\delta}\right)$.

Using automorphisms of $\boldsymbol{K} \times \boldsymbol{Z}_{q}$ we can change $\delta$. From Lemma 2.2 it is easy to see that exactly one image of $\delta$ under an automor- 
phism satisfies the conditions in the theorem.

THEOREM 2.4. The maximal equicontinuous factor of $\left(C_{n}, \phi_{F}\right)$ is isomorphic to $\left(\boldsymbol{K} \times \boldsymbol{Z}_{q}, M_{\dot{\delta}}\right)$ where $\delta$ is uniquely determined by the condition $\delta=(g, 1), g^{n} \neq 1$ for $n \neq 0$ and $g=\exp (\theta i)$ where $\theta \in(0, \pi / q)$.

Proof. By Theorem 2.3, it is sufficient to show that the maximal equicontinuous factor of $\left(C_{n}, \phi_{F}\right)$ is a one-dimensional manifold. Let $(Y, \psi)$ be the maximal equicontinuous factor of $\left(C_{n}, \phi_{F}\right)$ and let $p$ denote the canonical projection of $\left(C_{n}, \phi_{F}\right)$ onto $(Y, \psi)$. There exists a homomorphism $\pi^{\prime}$ of $\left(C_{n}, \phi_{F}\right)$ onto $\left(K, M_{r(\phi)}\right)$ and hence a homomorphism $\rho$ of $(Y, \psi)$ onto $\left(K, M_{r(\phi)}\right)$ such that $\pi^{\prime}=\rho \circ p$. The homomorphism $\pi^{\prime}=p^{\prime} \circ \pi$ where $\pi$ is the projection of $C_{n}$ onto $C$ and $p^{\prime}(x)=$ $p^{\prime}(y)$ if and only if $x$ and $y$ are proximal in $(C, \phi)$. Consequently if $x$ is a distal point of $(C, \phi)$, then $p^{-1}(p(x, i)) \subset\{(x, i): i=1, \cdots, n\}$. Let $E_{0}[x, i]=p^{-1}(p(x, i)) \cap\{(x, i): i=1, \cdots, n\}$ for any $x$ in $C$. Since each $E_{0}[x, i]$ consists of points which are distal, $\xi$ an element of the enveloping semigroup of $\left(C_{n}, \phi_{F}\right)$ will be injective on $E_{0}[x, i]$ and it is easy to check that $\xi\left(E_{0}[x, i]\right) \subset E_{0}[\xi(x, i)]$. It follows by using minimality that

$$
\left|E_{0}[x, i]\right|=\left|E_{0}[y, j]\right|
$$

for all $x, y \in C$ and $1 \leqq i, j \leqq n$.

Let $x_{0}$ be a distal point of $(C, \phi)$. To show that $Y$ is a one-dimensional manifold it suffices to show that $p\left(x_{0}, 1\right)$ has an open neighborhood homeomorphic to $(-1,1)$. Let $I$ be an open interval in $\boldsymbol{K}$ with end points in $T(\phi)$ such that $\pi^{\prime}\left(x_{0}, 1\right) \in I$. Let $V=p^{\prime-1}(I) \times\left\{i:\left(x_{0}, i\right) \in\right.$ $\left.E_{0}\left[x_{0}, 1\right]\right\}$. For sufficently small $V, p^{-1}(p(V))=V$ and $p(V)$ is open in $Y$. Let $R$ be the restriction of the equicontinuous structure relation of $\left(C_{n}, \phi_{F}\right)$ to $V$. It is immediate that $V / R$ is homeomorphic to $p(V)$. Because $\left|E_{0}[x, i]\right|$ is a constant $V / R$ is also homeomorphic to $\pi^{\prime}(V)=$ $I$ which completes the proof.

3. Some general lemmas. Through out this section $(X, T)$ will be a minimal transformation group and $E$ will denote its equicontinuous structure relation. Let $E[x]=\{y:(x, y) \in E\}$ and let $D$ be the set of distal points of $(X, T)$.

Lemma 3.1. If $E\left[x_{1}\right] \subset D$ for some $x_{1}$ in $X$ and $\left|E\left[x_{2}\right]\right|$ is finite for some $x_{2}$ in $X$, then

(a) $E\left[x^{\prime}\right] \subset D$ if and only if $\left|E\left[x^{\prime}\right]\right|=\min \{|E[x]|: x \in X\}$ and

(b) the canonical projection $p$ of $X$ onto $X / E$ is open at $x$ if and only if $x \in D$. 
Proof. (a) Let $\xi$ be an element of the enveloping semigroup of $(X, T)$. Then $\xi$ maps $E[x]$ into $E[\xi x]$. Since $\xi$ is injective on $D$, $|E[x]| \leqq|E[\xi x]|$ when $E[x] \subset D$. Hence $|E[x]|=\min \{\mid E[x]: x \in X\}$ whenever $E[x] \subset D$. If $E[x] \not \subset D$, then there exists $x^{\prime}, x^{\prime \prime} \in E[x]$ which are proximal. Choose $\xi$ so that $\xi x_{1}=x^{\prime}$. Then $x^{\prime \prime}$ is not in the range of $\xi$ and $\left|E\left[x_{1}\right]\right|<|E[x]|$.

(b) Use Proposition 2.3 [8].

The transformation group $(X, T)$ is locally proximally equicontinuous if for every $x$ in $X$ there exists a neighborhood $U$ of $x$ such that

$$
E \cap U \times U=P \cap U \times U
$$

where $P$ denotes the proximal relation. Note that if $U$ satisfies this equation then so does $t U$ where $t \in T$ and $V$ when $V \subset U$. Hence for minimal transformation groups it suffices to check it at one point. Clearly this is a weaker notion then proximal equicontinuity [1].

Lemma 3.2. The transformation group $(X, T)$ is locally proximally equicontinuous if and only if there exists $\varepsilon>0$ such that $(x, y) \in$ $E$ and $d(x, y)<\varepsilon$ implies $(x, y) \in P$.

We will denote the derived set of $A$, a subset of $X$, by $A^{\prime}$; i.e., $x \in A^{\prime}$ if and only if given $\varepsilon>0$ there exists $y \in A$ such that $0<$ $d(x, y)<\varepsilon$. As usual $\Delta=\{(x, x) \in X \times X\}$.

LEMMA 3.3. The following are equivalent:

(a) $P^{\prime} \backslash \Delta$ is a closed set in $X \times X$,

(b) there exist $\varepsilon>0$ such that

$$
P^{\prime} \cap\{(x, y): d(x, y)<\varepsilon\} \subset \Delta
$$

(c) given $x$ there exists an open neighborhood $U$ of $x$ such that $(P \cap U \times U)^{\prime} \subset \Delta$.

LEMMA 3.4. Let $R$ be a closed invariant equivalence relation on $X$ which is contained in $P$ and let $(Y, T)=(X / R, T)$. Then the following statements hold:

(a) If $(X, T)$ is locally proximally equicontinuous, then $(Y, T)$ is locally proximally equicontinuous.

(b) If $P_{X}^{\prime} / \Delta_{X}$ is closed, then $P_{Y}^{\prime} / \Delta_{Y}$ is closed.

Proof. (a) If $(Y, T)$ is not locally proximally equicontinuous, we can find $\left(y_{n}, y_{n}^{\prime}\right) \in E_{Y} \backslash P_{Y}$ such that $\left(y_{n}, y_{n}^{\prime}\right) \rightarrow(y, y)$. Let $\rho$ denote 
the canonical homomorphism of $(X, T)$ onto $(Y, T)$ and let $x_{n}, x_{n}^{\prime} \in X$ such that $\rho\left(x_{n}\right)=y_{n}$ and $\rho\left(x_{n}^{\prime}\right)=y_{n}^{\prime}$. We can assume that $\left(x_{n}, x_{n}^{\prime}\right)$ converges to $\left(x, x^{\prime}\right)$. Because $R \subset P$ it is clear that $\left(x_{n}, x_{n}^{\prime}\right) \in E_{x}$. Since $\rho(x)=y=\rho\left(x^{\prime}\right),\left(x, x^{\prime}\right) \subset R \subset P_{X}$. Let $U$ be an open set satisfying $E \cap U \times U=P \cap U \times U$. For some $\tau, \tau x, \tau x^{\prime} \in U$ and hence for large $n, \tau x_{n}, \tau x_{n}^{\prime} \in U$ and $\left(x_{n}, x_{n}^{\prime}\right) \in P_{X}$. Thus $\left(y_{n}, y_{n}^{\prime}\right) \in P_{Y}$ for large $n$ which is a contradiction.

(b) If $P_{Y}^{\prime} \backslash \Delta_{Y}$ is not closed, we can find $\left(y_{n}, y_{n}^{\prime}\right) \in P_{Y}^{\prime} \backslash \Delta_{Y}$ such that $\left(y_{n}, y_{n}^{\prime}\right) \rightarrow(y, y)$. Since given $\left(w_{1}, w_{2}\right) \in P_{Y}$, we can find $\left(z_{1}, z_{2}\right) \in P_{X}$ such that $\rho\left(z_{i}\right)=w_{i}$, we can find $\left(x_{n}, x_{n}^{\prime}\right) \in P_{X}^{\prime}$ such that $\rho\left(x_{n}\right)=y_{n}$ and $\rho\left(x_{n}^{\prime}\right)=y_{n}^{\prime}$. We can assume that $\left(x_{n}, x_{n}^{\prime}\right) \rightarrow\left(x, x^{\prime}\right)$. Then $\left(x, x^{\prime}\right) \in R \subset P_{X}$ and $\left(x, x^{\prime}\right) \in P_{X}^{\prime}$. Becuase $P_{X}^{\prime} \backslash \Delta_{X}$ is closed and $\left(x_{n}, x_{n}^{\prime}\right) \in P_{X}^{\prime}$ we see that $x \neq x^{\prime}$. But $P_{X}^{\prime} \backslash \Delta_{X}$ closed also implies that $P_{X}^{\prime} \cap P_{X} \subset \Delta_{X}$. Thus $x=x^{\prime}$ and we have a contradiction.

4. Locally circular minimal sets. A minimal cascade $(Y, \psi)$ will be called locally circular if there exists a minimal finite skew product $\left(C_{n}, \phi_{F}\right)$ of a circular cascade and a homomorphism $\theta$ of $\left(C_{n}, \phi_{F}\right)$ onto $(Y, \psi)$ such that $\theta(x)=\theta(y), x, y \in C_{n}$, implies $x$ and $y$ are doubly asymptotic. Locally circular is some what of a misnomer because they are not locally circular in the obvious meaning of this term. However, they can be characterized by using localizations of the properties which characterize circular cascades. This characterization is the following:

THEOREM 4.1. Let $(Y, \psi)$ be a minimal cascade on a compact metric space. Then $(Y, \psi)$ is locally circular if and only if the maximal equicontinuous factor of $(Y, \psi)$ is a one-dimensional manifold, $(Y, \psi)$ is locally proximally equicontinuous, and $P^{\prime} \backslash \Delta$ is closed subset of $Y \times Y$.

Proof. It follows from $\$ 2$ that minimal skew products of circular cascades satisfy the three conditions, and then by Lemma 3.4 so do locally circular cascades.

Now suppose $(Y, \psi)$ has the three properties stated in the theorem. Choose $\varepsilon>0$ such that $d(x, y)<\varepsilon$ and $(x, y) \in E$ implies $(x, y) \in P$ and such that $d(x, y)<\varepsilon$ and $(x, y) \in P^{\prime}$ implies $x=y$. If $1 /(n-1)<\varepsilon$, then $P \cap\{(x, y): 1 / n<d(x, y)<1 /(n-1)\}$ is finite. Therefore, $P \cap$ $\{(x, y): 0<d(x, y)<1 / N\}$ is countable where $1 / N<\varepsilon$, and $P \backslash \Delta$ is countable. Since $Y / E$ is uncountable, there exist points such that $E[y] \subset D$. Next we observe that $E[y]$ is finite for all $y$ in $Y$. If this were not the case we could find a $y$ and a sequence of distinct points $\left\{y_{n}\right\}$ such that $y_{n}$ converges to $y$ and $y_{n} \in E[y]$ for all $n$. For large $n$ and $m$ we would have $d\left(y_{n}, y_{m}\right)<\varepsilon / 2$ and hence $\left(y_{n}, y_{m}\right) \in P$. 
Holding $m$ fixed and letting $n$ go to infinity would show that $\left(y, y_{m}\right) \in$ $P^{\prime}$ and $d\left(y, y_{m}\right)<\varepsilon$. Hence we would have the contradiction $y=y_{m}$ for large $m$. Let $p$ be the canonical projection of $Y$ onto $Y / E$. By Lemma 3.1. $E\left[y^{\prime}\right] \subset D$ if and only if $\left|E\left[y^{\prime}\right]\right|=\min \{|E[y]|: y \in Y\}=$ $\alpha$ and $p$ is open at $y$ if and only if $y \in D$.

Since the maximal equicontinuous factor of $(Y, \psi)$ is a one-dimensional manifold, it has the form $\left(\boldsymbol{K} \times \boldsymbol{Z}_{m}, M_{\delta}\right)$ where $\delta=(h, 1)$ and $h^{n} \neq 1$ for $n \neq 0$ by Theorem 2.3. Let $\pi$ be the composition of $p$ and the projection of $\boldsymbol{K} \times \boldsymbol{Z}_{m}$ onto $\boldsymbol{K}$. The map $\pi$ is a homomorphism of $(Y, \psi)$ onto $\left(K, M_{h}\right)$. We now use a construction due to Veech [8, Theorem 3.1] to obtain minimal cascades $(X, \phi)$ and $(\tilde{X}, \tilde{\phi})$ and homomorphisms $q, \widetilde{q}$ and $\pi^{*}$ with the following properties:

(a) the diagram

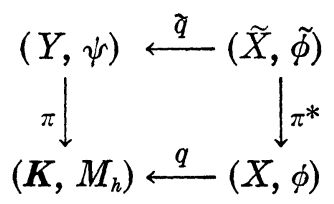

is commutative

(b) the map $\pi^{*}$ is open

(c) if $\pi$ is open at every point in $\pi^{-1}(z)$, then $q^{-1}(z)$ consists of exactly one point

(d) if $\pi$ is open at every point in $\pi^{-1}(\pi(y))$, then $\widetilde{q}^{-1}(y)$ consists of exactly one point.

The transformation group $(X, \phi)$ is constructed by taking the completion of $\left\{\pi^{-1}(z): \pi^{-1}(z) \subset D\right\}$ in the Hausdorff metric coming from $Y$. So a point $z^{*}$ of $X$ can also be thought of as a subset of $Y$. In this role we observe that $z^{*} \subset \pi^{-1}\left(q\left(z^{*}\right)\right)$ and $\left|z^{*}\right|=\alpha$. Choose $\varepsilon>0$ such that where $d(z, y)<\varepsilon$ the following hold:

$$
\begin{array}{lll}
(x, y) \in E & \text { implies } & (x, y) \in P \\
(x, y) \in P^{\prime} & \text { implies } & x=y \\
(x, y) \notin E & \text { implies } & \pi(x) \neq \pi(y) .
\end{array}
$$

Choose $\delta<\varepsilon / 2$ such that the open sets $S\left(y_{i}, \delta\right)=\left\{y: d\left(y, y_{i}\right)<\delta\right\}$ are disjoint where $\left\{y_{1}, \cdots, y_{n}\right\}=\pi^{1}(z) \subset D$ and $z$ is a fixed point of $\boldsymbol{K}$. Let $U=\bigcap_{i=1}^{n} \pi\left(S\left(y_{i}, \delta\right)\right)$ and $U_{i}=\pi^{-1}(U) \cap S\left(y_{i}, \delta\right)$. Clearly $\pi\left(U_{i}\right)=$ $U$. If $q\left(z^{*}\right) \in U$, then $z^{*} \cap U_{i}$ is a point. If $q\left(z^{*}\right)=q\left(w^{*}\right) \in U$, then $\left(z^{*} \cap U_{i}, w^{*} \cap U_{i}\right) \in P$. Suppose that $\left(z_{n}^{*}, w_{n}^{*}\right)$ is a sequence in $X \times X$ converging to $\left(z^{*} w^{*}\right)$ and $\left(z_{n}^{*}, w_{n}^{*}\right) \in P$. We have $q\left(z_{n}^{*}\right)=q\left(w_{n}^{*}\right)$ and $q\left(z^{*}\right)=q\left(w^{*}\right)$. We can assume $q\left(z^{*}\right) \in U$. If $z^{*} \neq w^{*}$, then $z^{*} \cap U_{i} \neq$ $w^{*} \cap U_{i}$ for some $i$. It is easy to see that $z_{n}^{*} \cap U_{i} \rightarrow z^{*} \cap U_{i}$ and $w_{n}^{*} \cap U_{i} \rightarrow w^{*} \cap U_{i}$. Therefore, $\left(z^{*} \cap U_{i}, w^{*} \cap U_{i}\right) \in P^{\prime}$ which contradicts 
$z^{*} \neq w^{*}$ because $d\left(z^{*} \cap U_{i}, w^{*} \cap U_{i}\right)<\varepsilon$. Hence for $(X, \phi), P^{\prime} \subset \Delta$ and $(X, \phi)$ is a circular cascade [5, Theorem 4.1]. If $(X, \phi)$ is transitive, then $(X, \phi)$ is equicontinuous, $q$ is an isomorphism, $(Y, \pi)$ is a covering space of $\boldsymbol{K},(Y, \psi)$ is isomorphic to some $\left(\boldsymbol{K} \times \boldsymbol{Z}_{k}, M_{\delta}\right)$ which is locally circular. If $(X, \phi)$ is intransitive, then $\pi^{*}$ is $n$ to one where $n=m \alpha=$ $\left|\widetilde{q}^{-1}\left(\pi^{-1}(z)\right)\right|$ if $\pi^{-1}(z) \subset D$. To finish the proof we must show that $\widetilde{q}\left(x_{1}\right)=\widetilde{q}\left(x_{2}\right)$ implies $x_{1}$ and $x_{2}$ are doubly asymptotic. If $\widetilde{q}\left(x_{1}\right)=\widetilde{q}\left(x_{2}\right)$, then either $\pi^{*}\left(x_{1}\right)=\pi^{*}\left(x_{2}\right)$ or $\pi^{*}\left(x_{1}\right), \pi^{*}\left(x_{2}\right)$ are proximal. The former is impossible because $\widetilde{q}$ maps $\pi^{*-1}\left(z^{*}\right)$ homeomorphically onto $z^{*}[8,219]$. (Here $z^{*}$ is first regarded as a point of $X$ and then as a subset of $Y$.) Let $A_{i}=\pi^{*-1}\left(\pi^{*}\left(x_{i}\right)\right)$. Then $A_{1} \cap A_{2}=\varnothing$. There exist $y \in A_{2}$ such that $x_{1}, y$ are positively \{negatively\} asymptotic. (Theorem 1.4 and Lemma 2.1.) We can assume that $\widetilde{q}\left(x_{1}\right), \widetilde{q}(y) \in U_{1}$ where $U_{1}, \cdots, U_{n}$ are as above. Then $\widetilde{q}(y), \widetilde{q}\left(x_{2}\right) \in U_{1} \cap z_{2}^{*}, z_{2}^{*}=\pi^{*}\left(x_{2}\right)$ implies $\widetilde{q}(y)=\widetilde{q}\left(x_{2}\right)$ and hence $y=x_{2}$. Therefore, $\left(x_{1}, x_{2}\right)$ are doubly asymptotic, and the proof is completed.

5. Invariants, Let $(Y, \psi)$ be a locally circular minimal set. From Theorem 2.4 we know that its maximal equicontinuous factor is of the form $\left(\boldsymbol{K} \times \boldsymbol{Z}_{q}, M_{\delta}\right)$ where $\delta=(h, 1)$. Moreover, $\delta$ is unique if we require that $h=e^{i \theta}$ where $0<\theta<\pi / q$. We call this $h$ the rotation number of $(Y, \psi)$ and denote it by $r(\psi)$. It is a well-defined invariant of $(Y, \psi)$. Clearly $q$ which is the number of components of the maximal equicontinuous factor is an invariant, which we denote by $c(\psi)$. By Lemma 3.1 we know that $|E[y]|$ where $E[y] \subset D$ is also a well-defined invariant of $(Y, \psi)$. We denote it by $e(\psi)$ and we let $n(\psi)=c(\psi) e(\psi)$. The positive integer $n(\psi)$ will be the canonical number of symbols in the permutation group. We also need a canonical circular cascade from which we can recapture $(Y, \psi)$ by taking a finite skew product with $n(\psi)$ symbols and identifying some doubly asymptotic points. A circular cascade $(X, \phi)$ is uniquely determined up to isomorphism by two invariants-the rotation number $r(\phi)$ and a subset $T(\phi)$ of $\boldsymbol{K}$ [5]. Certainly we want to have $r(\phi)=r(\psi)$. So we must have a way of using $(Y, \psi)$ to determine a suitable subset of $\boldsymbol{K}$. A slight modification of the original definition of $T(\phi)$ does this.

LEMMA 5.1. Let $\rho_{1}$ and $\rho_{2}$ be homomorphisms of $(Y, \psi)$ onto $\left(\boldsymbol{K}, M_{r\left(\psi_{1}\right)}\right)$. Then there exists $a w \in \boldsymbol{K}$ such that $\rho_{1}=M_{w} \circ \rho_{2}$.

Proof. Let $p$ be a canonical projection of $(Y, \psi)$ onto its maximal equicontinuous factor $\left(K \times Z_{q}, M_{\delta}\right)$ and let $\pi$ be the composition of $p$ and the projection of $\boldsymbol{K} \times \boldsymbol{Z}_{q}$ onto $\boldsymbol{K}$. It suffices to show that there exists $w \in \boldsymbol{K}$ such that $\pi=M_{w} \circ \rho_{1}$. There exists a homomorphism $\rho^{\prime}$ 
of $\left(\boldsymbol{K} \times Z_{q}, M_{\delta}\right)$ onto $\left(\boldsymbol{K}, M_{r(\psi)}\right)$ such that $\rho^{\prime} \circ p=\rho_{1}$. If we modify $\rho_{1}$ by a rotation we can assume $\rho^{\prime}$ is also a group homomorphism of $\boldsymbol{K} \times \boldsymbol{Z}_{q}$ onto $\boldsymbol{K}$. In other words, there exists $w \in \boldsymbol{K}$ and a group homomorphism of $\boldsymbol{K} \times \boldsymbol{Z}_{q}$ onto $\boldsymbol{K}$ such that $M_{w} \circ \rho_{1}=\rho^{\prime} \circ p$ and $\rho^{\prime} \circ M_{\delta}=$ $M_{r(\psi(\psi)} \circ \rho^{\prime}$. So it suffices to show that $\rho^{\prime}(z, i)=z$.

It is easy to see that $\rho^{\prime}(z, i)=\xi^{i} z^{n}$ for some $n \in Z$ and $\xi$ a $q$ th root of unity. We also have $\rho^{\prime}(\delta)=\rho^{\prime}(r(\psi), 1)=r(\psi)$, and hence we have $\xi r(\psi)^{n}=r(\psi)$. If $n \neq 1, r(\psi)$ is a root of unity which is impossible because $r(\psi)$ is a generator of $K$ (Theorem 2.4). If $n=1, \xi=$ 1 and $\rho^{\prime}(z, i)=z$.

Let $\rho$ be any homomorphism of $(Y, \psi)$ onto $\left(\boldsymbol{K}, M_{r(\psi)}\right)$ and let $T(\psi)=\left\{z \in K: \rho^{-1}(z) \subset D\right\}$. Suppose $\theta$ is an isomorphism $(Y, \psi)$ onto $\left(Y^{\prime}, \psi^{\prime}\right)$ and $\rho^{\prime}$ is a homomorphism of $\left(Y^{\prime}, \psi^{\prime}\right)$ onto $\left(K, M_{r\left(\psi^{\prime}\right)}\right)$. (We already know that $r(\psi)=r\left(\psi^{\prime}\right)$.) Applying Lemma 5.1 to $\rho_{1}=\rho$ and $\rho_{2}=\rho^{\prime} \circ \theta$ we obtain $w \in \boldsymbol{K}$ such that $\rho=M_{w} \circ \rho^{\prime} \circ \theta$. Since $\rho^{-1}(z)=$ $\theta^{-1}\left[\rho^{\prime-1}\left(w^{-1} z\right)\right], \rho^{-1}(z)$ consists of distal points if and only if $\rho^{-1}\left(w^{-1} z\right)$ consists of distal points. Therefore, $\left\{z: \rho^{-1}(z) \subset D\right\} \equiv\left\{z: \rho^{\prime-1}(z) \subset D\right\}$ and $T(\psi)$ is a well defined invariant. In addition, $T(\psi)$ is invariant under $M_{r(\psi)}$ and $\boldsymbol{K} \backslash T(\psi)$ is countable. Hence $r(\psi)$ and $T(\psi)$ uniquely determine a circular cascade. Note that if $(Y, \psi)=\left(C_{n}, \dot{\phi}_{F}\right)$ where $(C, \phi)$ is a circular cascade and $F: C \rightarrow S_{n}$ continuously, it is not true in general that $r(\psi)=r(\phi), T(\psi)=T(\phi)$ and $n(\psi)=n$.

Next we want to connect our invariants with the circular cascade $(X, \phi)$ and the finite skew product $(\tilde{X}, \tilde{\phi})$ which we constructed in the proof of Theorem 4.1. The starting point for the construction was any homomorphism $p$ of $(Y, \psi)$ onto $\left(K \times Z_{q}, M_{\delta}\right), \delta=(h, 1)$ such that $p(x)=p(y)$ if and only if $(x, y) \in E$. In this section we will always assume that $\delta=(r(\psi), 1)$. Let $\rho$ be any homomorphism of $(Y, \psi)$ onto $\left(K, M_{r\left(\psi^{\prime}\right)}\right)$. It follows from Lemma 5.1 that we can find $p$ as above such that $\rho$ equals the composition of $p$ and the projection of $\boldsymbol{K} \times \boldsymbol{Z}_{q}$ onto $K$. Consequently if we apply the Veech construction to any $\rho:(Y, \psi) \rightarrow\left(\boldsymbol{K}, M_{r(\psi)}\right)$ we will always get a circular cascade $(X, \phi)$ and a finite skew product $(\widetilde{X}, \widetilde{\phi})$ of $(X, \phi)$ using $n(\psi)$ symbols. The homomorphisms $q, \widetilde{q}$ and $\rho^{*}$ satisfy conditions (a), (b), (c), and (d) in the proof of Theorem 4.1 and $\widetilde{q}(x)=\widetilde{q}(y)$ implies $x$ and $y$ are doubly asymptotic.

THEOREM 5.2. Let $(Y, \psi)$ be a locally circular minimal set and let $\rho$ be a homomorphism of $(Y, \psi)$ onto $\left(K, M_{r(\psi)}\right)$. Suppose $(X, \phi)$ and $(\widetilde{X}, \tilde{\phi})$ are the minimal cascades and $q, \widetilde{q}$, and $\rho^{*}$ are the homomorphisms obtained from Veech's construction [8, Theorem 3.1]. Then the following statements are true:

(a) The minimal set $(X, \phi)$ is a circular cascade, $(\tilde{X}, \tilde{\phi})$ is a finite skew product of $(X, \phi)$ with $n(\psi)$ symbols, and $\widetilde{q}(x)=\widetilde{q}(y)$ implies $x$ 
and $y$ are doubly asymptotic.

(b) The invariants are related by the equations

$$
\begin{gathered}
r(\psi)=r(\tilde{\phi})=r(\phi) \\
T(\psi)=T(\tilde{\phi})=T(\dot{\phi}) \\
n(\psi)=n(\tilde{\phi}) .
\end{gathered}
$$

(c) The minimal sets $(X, \phi)$ and $(\tilde{X}, \tilde{\phi})$ are uniquely determined up to isomorphism by the isomorphism class of $(Y, \psi)$.

(d) If $(Y, \psi)$ is a finite skew product of a circular cascade, then $\tilde{q}$ is an isomorphism of $(\tilde{X}, \tilde{\phi})$ onto $(Y, \psi)$.

Proof. (a) This was established in the preceding paragraph.

(b) Because $\widetilde{q}(x)=\widetilde{q}(y)$ implies $x$ and $y$ are doubly asymptotic, $(Y, \psi)$ and $(\tilde{X}, \tilde{\phi})$ have the same maximal equicontinuous factor and we can find a homomorphism $\rho^{\prime}$ such that $\rho^{\prime}=\rho \circ \widetilde{q}$. It follows that $r(\psi)=r(\tilde{\phi}), T(\psi)=T(\tilde{\phi})$, and $n(\psi)=n(\tilde{\phi})$.

Since there are points such that $\left|q^{-1}(z)\right|=1$, it follows that $q(x)=$ $q(y)$ if and only if $x$ and $y$ are proximal. Thus $r(\psi)=r(\phi)$. Using Lemma 3.1 we see that $\rho^{-1}(z) \subset D$ if and only if $\left|q^{-1}(z)\right|=1$ which implies $T(\psi)=T(\phi)$.

(c) Let $\theta$ be an isomorphism of between the locally circular cascades $\left(Y_{1}, \psi_{1}\right)$ and $\left(Y_{2}, \psi_{2}\right)$, and let $\rho_{i}$ be a homomorphism of $\left(Y_{i}, \psi_{i}\right)$ onto $\left(\boldsymbol{K}, M_{h}\right)$ where $r\left(\psi_{1}\right)=h=r\left(\psi_{2}\right)$. We must find isomorphisms $\hat{\theta}$ and $\tilde{\theta}$ to complete the following commutative diagram:

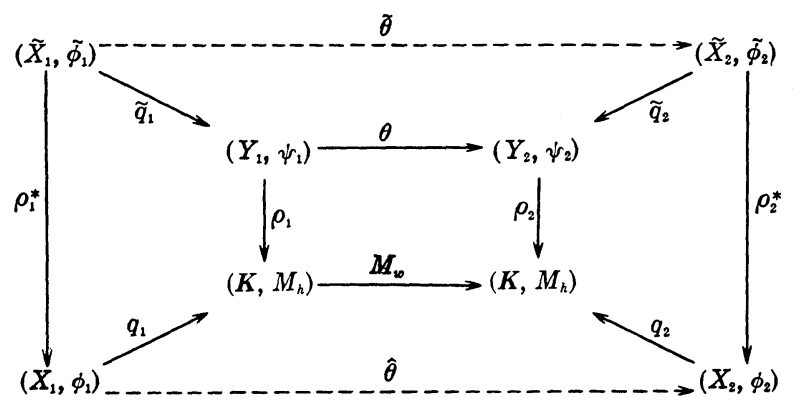

The $M_{w}$ is obtained by applying Lemma 5.1. The existence of $\hat{\theta}$ follows from Theorem 2.2 of [5]. Examining Veech's construction one sees that $\tilde{X}$ is the closure of $\left\{\left(y, \rho^{-1}[\rho(y)]\right): \rho^{-1}[\rho(y)] \subset D\right\}$ in $Y \times Y^{Y}$ where the metric is the metric on $Y$ plus associated Hausdorff metric and $\tilde{\phi}(y, A)=(\phi(y), \phi(A))$. Finally let $\widetilde{\theta}$ be the obvious map.

(d) This follows because the projection of a finite skew product of a circular cascade is either $e(\psi)$ or $2 e(\psi)$ to one and hence $\rho$ is either $n(\psi)$ or $2 n(\psi)$ to one. 
Let $(X, \phi)$ be a fixed circular cascade, let $n$ be a positive integer, and let $C\left(X, S_{n}\right)$ denote the continuous functions from $X$ into $S_{n}$. We say two functions $F$ and $G$ in $C\left(X, S_{n}\right)$ are cohomologous if there exists $a \in C\left(X, S_{n}\right)$ such that for all $x$ in $X$

$$
F(x)=a(\phi(x))^{-1} G(x) a(x) .
$$

It is easy to see that this is an equivalence relation on $C\left(X, S_{n}\right)$. We will call the equivalence classes cohomology classes of $(X, \phi)$ for $S_{n}$. If $F$ and $G$ are cohomologous, then $\theta(x, i)=(x, a(x) i)$ is an isomorphism of $\left(X_{n}, \phi_{F}\right)$ onto $\left(X_{n}, \phi_{G}\right)$. To obtain the converse of this we need a slightly weaker notion. One can see this by considering $F$ and $G=F \circ \beta$ where $\beta$ is an automorphism. Here $\theta(x, i)=\left(\beta^{-1}(x), i\right)$ is an isomorphism of $\left(X_{n}, \phi_{F}\right)$ onto $\left(X_{n}, \phi_{G}\right)$, but if $\beta$ is properly chosen there is no isomorphism of $\left(X_{n}, \phi_{F}\right)$ onto $\left(X_{n}, \phi_{G}\right)$ which leaves the $x$ coordinate fixed. We will say $F$ and $G$ are weakly cohomologous if there exists an automorphism $\beta$ of $(X, \phi)$ such that $F$ and $G \circ \beta$ are cohomologous. This is also an equivalence relation on $C\left(X, S_{n}\right)$.

THEOREM 5.3. Let $(X, \phi)$ be a circular cascade and let $F, G \in$ $C\left(X, S_{n}\right)$ such that $\left(X_{n}, \phi_{F}\right)$ and $\left(X_{n}, \phi_{G}\right)$ are minimal. Then $\left(X_{n}, \phi_{F}\right)$ is isomorphic to $\left(X_{n}, \phi_{G}\right)$ if and only if $F$ and $G$ are weakly cohomologous.

Proof. First suppose that $F$ and $G$ are weakly cohomologous. So there exists $a \in C\left(X, S_{n}\right)$ and $\beta$ an automorphism such that $F(x)=a(\phi(x))^{-1} G(\beta(x)) a(x)$. Define $\theta$ by $\theta(x, i)=(\beta(x), a(x) i)$. Then $\theta \circ \phi_{F}(x, i)=\theta(\phi(x), F(x) i)=(\beta(\phi(x)), a(\phi(x)) F(x) i)=(\phi(\beta(x)), G(\beta(x)) a(x) i)=$ $\phi_{G}(\beta(x), a(x) i)=\phi_{G} \circ \theta(x, i)$. Clearly $\theta$ is a homeomorphism. Hence $\left(X_{n}, \phi_{F}\right)$ and $\left(X_{n}, \phi_{F}\right)$ are isomorphic.

Now suppose that $\theta$ is an isomorphism of $\left(X_{n}, \phi_{F}\right)$ onto $\left(X_{n}, \phi_{G}\right)$. It follows that their maximal equicontinuous factors are isomorphic. Let $\left(\boldsymbol{K} \times \boldsymbol{Z}_{q}, M_{\delta}\right)$ denote their maximal equicontinuous factor where $\delta=(h, 1), h=\exp (\alpha i), \alpha \in(0, \pi / q)$. We have the following commutative diagram:

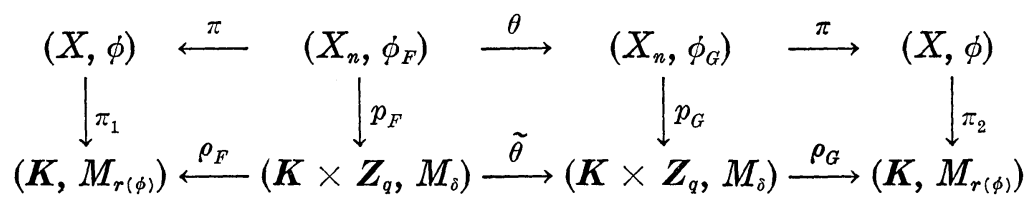

where $\pi(x, i)=x, p_{F}, p_{G}, \pi_{1}$, and $\pi_{2}$ are canonical projections onto maximal equicontinuous factors, and $\rho_{F}, \rho_{G}$, and $\tilde{\theta}$ are induced by $\pi_{1} \circ \pi, \pi_{2} \circ \pi$ and $p_{G} \circ \theta$. By changing $p_{G}$ by a rotation we can assume that $\tilde{\theta}$ is the identity. (If $\tilde{\theta}(1,0)=(1,0)$, then $\tilde{\theta}$ is an automorphism 
of the topological group $\boldsymbol{K} \times \boldsymbol{Z}_{q}$ and the identity is the only automorphism which leaves $\delta$ fixed.) By changing $\pi_{1}$ and $\pi_{2}$ by rotations we can assume that $\rho_{F}$ and $\rho_{G}$ are group homomorphisms. Since $\rho_{F}(z, 0)=$ $z^{k}$ for some $k \in Z$ and $\rho_{F}(1,1)=\xi$, a $q$ th root of unity, we have $\rho_{F}(z, i)=z^{k} \xi^{i}$. It is easy to see that $\rho_{F}$ is $|k| q$ to one. Suppose $x$ is a distal point of $(X, \phi)$. Then $\pi_{1}^{-1}\left(\pi_{1}(x)\right)=x$ and $\pi^{-1}(x)=\left(\pi_{1} \circ \pi\right)^{-1}(z)$ consists of $n$-distal points where $z=\pi_{1}(x)$. Moreover, $\left(\pi_{1} \circ \pi\right)^{-1}(z)$ is saturated with respect to the equicontinuous structure relation of $\left(X_{n}, \phi_{F}\right)$ and hence $\rho_{F}$ is $e\left(\phi_{F}\right)$ to one on it. But $\left(\pi_{1} \circ \pi\right)^{-1}(z)=p_{F}^{-1}\left(\rho_{F}^{-1}(z)\right)$ and hence $|k| q e\left(\phi_{F}\right)=n$ and $|k|=n / q e\left(\phi_{F}\right)=n / c\left(\phi_{F}\right) e\left(\phi_{F}\right)=n / n\left(\phi_{F}\right)$. Similarly $\rho_{G}(z, i)=z^{l} \eta^{i}$ where $\eta$ is a $q$ th root of unity and $|l|=n / n\left(\phi_{G}\right)=n / n\left(\phi_{F}\right)=$ $|k|$. Since $\rho_{F}(\delta)=r(\phi)=\rho_{G}(\delta), \xi h^{k}=\eta h^{k}$ or $\eta h^{-k}$. If $\xi h^{k}=\eta h^{-k}$, then $h$ is a $2 k q$ th root of unity which is impossible. If $\xi h^{k}=\eta h^{k}$, then $\xi=\eta$ and $\rho_{F}=\rho_{G}$.

We can write $\theta(x, i)=\left(\theta_{1}(x, i), \theta_{2}(x, i)\right)$ where $\theta_{1}$ and $\theta_{2}$ are continuous. We will show that $\theta_{1}(x, i)=\theta_{1}(x, j)$ for all $x, i$ and $j$. Suppose $x$ is a distal point in $(X, \phi)$. Then $\pi^{-1}(x)=\{x\} \times\{1, \cdots, n\}$ consists of $n$ points and so does $\theta\left(\pi^{-1}(x)\right)$. Now $\theta\left(\pi^{-1}(x)\right)=\theta\left(\pi_{1} \circ \pi^{-1}(z)\right)=$ $\theta\left[\left(\rho_{F} \circ p_{F}\right)^{-1}(z)\right]=\theta\left(p_{F}^{-1}\left[\rho_{F}^{-1}(z)\right]=p_{G}^{-1}\left[\rho_{F}^{-1}(z)\right]=p_{G}^{-1}\left[\rho_{G}^{-1}(z)\right]=\left(\rho_{G} \circ p_{G}\right)^{-1}(z)=\right.$ $\left(\pi_{2} \circ \pi\right)^{-1}(z)=\pi^{-1}\left[\pi_{2}^{-1}(z)\right]$. From cardinality considerations $\pi_{2}^{-1}(z)$ is a point. Therefore, $\theta\left(\pi^{-1}(x)\right)=\left\{x^{\prime}\right\} \times\{1,2, \cdots, n\}$ where $x^{\prime}=\pi_{2}^{-1}(z)$ for all distal points $x$. In other words, if $x$ is distal $\theta_{1}(x, i)=\theta_{1}(x, j)$ for all $i$ and $j$. Since the distal points are dense in $X$ and $\theta_{1}$ is continuous, we obtain $\theta_{1}(x, i)=\theta_{1}(x, j)$ for all $x$, $i$, and $j$. We can now write $\theta_{1}(x, i)=\theta_{1}(x)$. For each $x, a_{1}(x) i=\theta_{2}(x, i)$ defines an element of $S_{n}$, and $a_{1} \in C\left(X, S_{n}\right)$ because $\theta_{2}$ is continuous. The equation $\phi_{G} \circ \theta=$ $\theta \circ \phi_{F}$ can be written

$$
\left(\phi\left[\theta_{1}(x)\right], G\left(\theta_{1}(x)\right) a_{1}(x) i\right)=\left(\theta_{1}[\phi(x)], a_{1}(\phi(x)) F(x) i\right) .
$$

Therefore, $\phi \circ \theta_{1}=\theta_{1} \circ \phi$ and $G\left(\theta_{1}(x)\right) a_{1}(x)=a_{1}(\phi(x)) F(x)$ which completes the proof.

CoROllary 5.4. Let $(X, \phi)$ be a circular cascade and let $F$ and $G$ be elements of $\boldsymbol{C}\left(X, S_{n}\right)$ such that $\left(X_{n}, \phi_{F}\right)$ and $\left(X_{n}, \phi_{G}\right)$ are minimal. Then $F$ and $G$ are cohomologous if and only if there exists an isomorphism $\theta:\left(X_{n}, \phi_{F}\right) \rightarrow\left(X_{n}, \phi_{G}\right)$ of the form $\theta(x, i)=\left(x, \theta_{2}(x, i)\right)$.

\section{Proof. Obvious.}

It is clear that an isomorphism between two minimal sets induces a bijection between their weak cohomology classes, and it follows from Theorem 5.3 that two different isomorphisms induce the same bijection. For two weak cohomology classes $W_{1}$ and $W_{2}$ we will write 
$W_{1}=W_{2}$ provided the corresponding ranges are equal, the domains are isomorphic, and $W_{1}$ is carried onto $W_{2}$ by the induced map.

Let $(Y, \psi)$ be a locally circular cascade and let $\rho$ be a homomorphism of $(Y, \psi)$ onto $\left(\boldsymbol{K}, M_{r(\psi)}\right)$. Now construct the circular cascade $(X, \phi)$ and the finite skew product $(\widetilde{X}, \tilde{\phi})$ as above. There exists $F \in$ $\boldsymbol{C}\left(X, S_{n(\psi)}\right)$ such that $(\tilde{X}, \tilde{\phi})$ is isomorphic to $\left(X_{n(\psi)}, \dot{\phi}_{F}\right)$. Let $W(\psi)$ be the weak cohomology class of $F$. It follows easily from Theorems 5.2 and 5.3 that $W(\psi)$ is an invariant of $(Y, \psi)$. The invariants $n(\psi)$, $r(\psi), T(\psi)$, and $W(\psi)$ do not uniquely determine $(Y, \psi)$. However, we will show that they do uniquely determine $(\widetilde{X}, \tilde{\phi})$, which is itself and invariant of $(Y, \psi)$. In other words, we can classify the minimal finite skew products of circular cascades and hence the locally circular ones except for the identification of some doubly asymptotic orbits.

Theorem 5.5. Let $\left(Y_{1}, \psi_{1}\right)$ and $\left(Y_{2}, \psi_{2}\right)$ be two minimal finite skew products of circular cascades. Then $\left(Y_{1}, \psi_{1}\right)$ and $\left(Y_{2}, \psi_{2}\right)$ are isomorphic if and only if

$$
\begin{aligned}
n\left(\psi_{1}\right) & =n\left(\psi_{2}\right) \\
r\left(\psi_{1}\right) & =r\left(\psi_{2}\right) \\
T\left(\psi_{1}\right) & =T\left(\psi_{2}\right) \\
W\left(\psi_{1}\right) & =W\left(\psi_{2}\right) .
\end{aligned}
$$

Proof. If they are isomorphic, then the equations hold because in the course of this section we have established that $n\left(\psi_{i}\right), r\left(\psi_{i}\right), T\left(\psi_{i}\right)$, and $W\left(\psi_{i}\right)$ depend only on the isomorphism class of $\left(Y_{i}, \psi_{i}\right)$.

Assume the equations hold. Let $(X, \phi)$ be a circular cascade determined by $r\left(\psi_{1}\right)=r\left(\psi_{2}\right)$ and $T\left(\psi_{1}\right) \equiv T\left(\psi_{2}\right)$. Then $W=W\left(\psi_{1}\right)=$ $W\left(\psi_{2}\right)$ can be realized in $\boldsymbol{C}\left(X, S_{n}\right)$ where $n=n\left(\psi_{1}\right)=n\left(\psi_{2}\right)$ (Theorem 5.2). Let $F \in W$. Then $\left(Y_{1}, \psi_{1}\right)$ and $\left(Y_{2}, \psi_{2}\right)$ are both isomorphic to $\left(X_{n}, \phi_{F}\right)$.

6. Normal representatives of cohomology classes. Let $(C, \phi)$ denote a fixed circular cascade where $C$ is a Cantor set in $K$ and $\phi$ is the restriction of a homeomorphism of $\boldsymbol{K}$ without periodic points to its unique minimal subset $C$. Observe that if $(a, b)$ is a complementary interval of $C$, then so is $(\phi(a), \phi(b))$. A cohomology class $\mathscr{C}$ is minimal if $F \in \mathscr{C}$ implies $\left(C_{n}, \dot{\phi}_{F}\right)$ is minimal. We want to investigate the minimal cohomology classes of $(C, \phi)$ for the group $S_{n}$. (Note that we already know that there is only one minimal cohomology class of $\left(K, M_{g}\right)$ for the group $S_{n}$.)

Definition. Let $F: C \rightarrow S_{n}$ continuously and let $\left(a_{i}, b_{i}\right), i=1, \cdots, p$ denote the complementary intervals of $C$ such that $F\left(a_{i}\right) \neq F\left(b_{i}\right)$. We 
say $F$ is normal if for $n>0$ and $1 \leqq i, j \leqq p$

$$
\left(\phi^{n}\left(a_{i}\right), \phi^{n}\left(b_{i}\right)\right) \neq\left(a_{j}, b_{j}\right)
$$

with at most one exception of the form

$$
\left(\phi\left(a_{i}\right), \phi\left(b_{i}\right)\right)=\left(a_{j}, b_{j}\right), \quad i \neq j \text {. }
$$

Let $\mathscr{C}$ be a cohomology class of $(C, \phi)$ for $S_{n}$. We say $F$ is a normal representative of $\mathscr{C}$ if $F$ is in $\mathscr{C}$ and $F$ is normal. One of the advantages of normality is the existence of a simple test for minimality.

THEOREM 6.1. Let $F$ be normal. Then $\left(C_{n}, \phi_{F}\right)$ is minimal if and only if the subgroup of $S_{n}$ generated by the range of $F$ acts transitively on $\{1, \cdots, n\}$.

Proof. The subgroup of $H$ of $S_{n}$ generated by the range of $F$ consists of all finite products of the form $F\left(x_{1}\right) F\left(x_{2}\right) \cdots \cdots F\left(x_{n}\right)$. It is clear that if $\left(C_{n}, \phi_{F}\right)$ is minimal, then $H$ acts transitively on $\{1, \cdots, n\}$. (This observation does not require the normality of $F$. Just examine the orbit closure of any point in $\left(C_{n}, \phi_{F}\right)$.)

Suppose $F$ is normal and $H$ acts transitively on $\{1, \cdots, n\}$. If for each $i, 1 \leqq i \leqq n, C \times\{i\}$ is contained in one minimal set of $\left(C_{n}, \phi_{F}\right)$, then $C \times\{i\}$ and $C \times\{F(x) i\}$ are in the same minimal set for all $x$ in $C$. Consequently it suffices to prove that $C \times\{i\}$ is contained in one minimal subset of $\left(C_{n}, \phi_{F}\right)$. This is where the normality of $F$ is needed. Let $\left(a_{j}, b_{j}\right) j=1, \cdots, p$ be the complementary intervals of $C$ such that $F\left(a_{i}\right) \neq F\left(b_{i}\right)$. If the allowed exception occurs, we can assume by renumbering that

$$
\left(\phi\left(a_{2}\right), \phi\left(b_{2}\right)\right)=\left(a_{1}, b_{1}\right) .
$$

Let $(a, b)$ be any complementary interval of $C$ except $\left(a_{1}, b_{1}\right)$. Then it is clear that $\phi_{F}^{m}(a, i)$ and $\phi_{F}^{m}(b, i)$ belong to the same $C \times\{j(m)\}$ either for all $m>0$ or for all $m \leqq 0$ when $1 \leqq j(m) \leqq n$ because $F$ is normal. Therefore, they are asymptotic in one sense and in the same minimal set. Since the minimal sets of $\left(C_{n}, \phi_{F}\right)$ are open and closed in $C_{n}$, it follows that, for each $i, C \times\{i\}$ is contained in one minimal subset of $\left(C_{n}, \phi_{F}\right)$.

We will show that every minimal cohomology class has a normal representative and that the complementary intervals $\left(a_{i}, b_{i}\right)$ can be specified. Let $F$ be a fixed continuous map from $C$ to $S_{n}$ such that $\left(C_{n}, \phi_{F}\right)$ is minimal. We will proceed by taking $C_{n}$ apart and reassemblying it.

DEFINITION. A finite collection of complementary intervals will be called admissible if they satisfy the following conditions: 
1. If $F(a) \neq F(b)$ for some complementary interval $(a, b)$, then $(a, b)$ is in $\mathscr{A}$.

2. If $(a, b)$ and $\left(\phi^{n}(a), \phi^{n}(b)\right)$ are in $\mathscr{A}$ and $n>0$, then $\left(\phi^{k}(a), \phi^{k}(b)\right)$ is in $\mathscr{A}$ for $k=1, \cdots, n-1$.

Henceforth, $\mathscr{A}$ will be an admissible collection of complementary intervals. We will also assume that $\mathscr{A}=\left\{\left(a_{1}, b_{1}\right), \cdots,\left(a_{q}, b_{q}\right)\right\}$ is indexed counterclockwise and $\left(a_{1}, b_{1}\right)$ has been chosen so that $\left(\phi^{n}\left(a_{1}\right), \phi^{n}\left(b_{1}\right)\right) \notin$ $\mathscr{A}$ for all $n>0$. Let $I_{i}=\left[b_{i}, a_{i+1}\right] \cap C$ if $i<q$ and $I_{q}=\left[b_{q}, a_{1}\right] \cap C$. Define $L_{1}$ to be the identity element of $S_{n}$ and $L_{i}=A_{-}\left(a_{i}, b_{i}\right) L_{i-1}$. Now let

$$
\mathrm{M}_{j}=\bigcup_{i=1}^{q} I_{i} \times\left\{L_{i} j\right\}
$$

The proof of the next lemma is straight forward and is left to the reader.

LEMMA 6.2. The following statements are valid:

(a) $M_{i} \cap M_{j}=\varnothing$ when $i \neq j$.

(b) $C_{n}=\mathrm{U}_{i=1}^{n} M_{i}$.

(c) The map $\theta$ defined by $\theta(x, k)=(x, j)$ if $(x, k) \in M_{j}$ is a homeomorphism of $C_{n}$ onto $C_{n}$.

The next step is to determine $G: C \rightarrow S_{n}$ such that $\theta \circ \phi_{F} \circ \theta^{-1}=\phi_{G}$ and then investigate the intervals on which $G$ is constant. From Corollary 5.4 we know that $F$ and $G$ are cohomologous. So $G$ is a representative of the cohomology class of $F$. Define $G$ by $G(x) i=j$ if $\phi_{F}\left(\theta^{-1}(x, i)\right) \in M_{j}$. It is easy to check that $G(x)$ is injective on $\{1, \cdots, n\}$ and hence an element of $S_{n}$. If $\phi_{F}\left(\theta^{-1}(x, i)\right) \in M_{j}$, then $G(x) i=$ $j$ and $\theta \circ \phi_{F} \circ \theta^{-1}(x, i)=(\phi(x), j)=(\phi(x), G(x) i)=\phi_{G}(x, i)$. Therefore, $\theta \circ \phi_{F} \circ \theta^{-1}=\phi_{G}$. Notice we did not establish the continuity of $G$, but this follows from the continuity of $\phi_{G}$ which we know because $\phi_{G}=$ $\theta \circ \phi_{F} \circ \theta^{-1}$.

Lemma 6.3. The function $G$ constructed as above is normal.

Proof. We will present only an outline of the proof leaving the details to the reader.

The first step is to show that if $(a, b)$ is a complementary interval of $C$ such that $(a, b) \notin \mathscr{A}$ and $(\phi(a), \phi(b)) \notin \mathscr{A}$, then $G(a)=G(b)$.

Next suppose that $(a, b)$ is a complementary interval such that $(a, b) \notin \mathscr{A}$ and $\left(\phi^{m}(a), \phi^{m}(b)\right)=\left(a_{j(m)}, b_{j(m)}\right) \in \mathscr{A}$ where $j(m)=2, \cdots, p$ and $1 \leqq m \leqq N$. The goal here is to show that $G\left(\phi^{m}(a)\right)=G\left(\phi^{m}(b)\right)$ for $0 \leqq m \leqq N-1$. It suffices to show that $G(a, m)=G(b, m)$ for $0 \leqq m \leqq N$ because $G\left(\phi^{m}(x)\right)=G(x, m+1) G(x, m)^{-1}$. Observe that 
$G(x, m) k=k^{\prime}$ if and only if $\phi_{F}^{m} \circ \theta^{-1}(x, k) M_{k^{\prime}}$ and that $(a, i)$ and $(b, i)$ are negatively asymptotic for $1 \leqq i \leqq m$. This part can now be completed using the definition of $M_{k}$.

From the above it follows that $G(a) \neq G(b)$ implies $(a, b) \in \mathscr{A}$ and $(\phi(a), \phi(b)) \notin \mathscr{A}$ or $(\phi(a), \phi(b))=\left(a_{1}, b_{1}\right)$ by eliminating the other possibilities. In other words, $G$ is normal.

THEOREM 6.4. Every minimal cohomology class of $(C, \phi)$ for $S_{n}$ has a normal representative.

Proof. Let $\mathscr{C}$ be a minimal cohomology class of $(C, \dot{\phi})$ for $S_{n}$ and let $F \in \mathscr{C}$. Then clearly we can find an admissible collection of complementary intervals for $F$ and construct $G$ cohomologous to $F$ as above. By Lemma 6.3 $G$ is normal.

Let $F$ be a normal representative of $\mathscr{C}$, and let $J(F)=\left\{\left(a_{1}, b_{1}\right), \cdots\right.$, $\left.\left(a_{q}, b_{q}\right)\right\}$ be the complementary intervals of $C$ satisfying

(a) $F\left(a_{i}\right) \neq F\left(b_{i}\right), i=1, \cdots, q$.

(b) If $(a, b)$ is any complementary interval for which $F(a) \neq F(b)$, then either $(a, b)=\left(a_{i}, b_{i}\right)$ for some $i$ in $\{1, \cdots, q\}$ or $(\dot{\phi}(a), \dot{\phi}(b))=$ $\left(a_{1}, b_{1}\right)$ but not both.

(c) They are arranged counterclockwise in $C$. We will refer to $J(F)$ as the jumps of $F$ and when it happens that $F(a) \neq F(b)$ and $(\dot{\phi}(a), \dot{\phi}(b))=\left(a_{1}, b_{1}\right)$ we will say that $F$ has an exceptional jump at $(a, b)$.

THEOREM 6.5. Let $F$ be a normal representative of the minimal cohomology class $\mathscr{C}$. Let $\mathscr{A}=\left\{\left(c_{1}, d_{1}\right), \cdots,\left(c_{p}, d_{p}\right)\right\}$ be a collection of complementary intervals of $C$ numbered counterclockwise and satisfying the following conditions:

(a) $\phi\left(c_{i}, d_{i}\right) \neq\left(c_{j}, d_{j}\right)$ for all $n>0,1 \leqq i, j \leqq p$.

(b) For each $i$ there exists $n$ such that $F\left(\phi^{n}\left(c_{i}\right)\right) \neq F\left(\dot{\phi}^{n}\left(d_{i}\right)\right)$.

(c) For each complementary interval $(a, b)$ such that $F(a) \neq F(b)$, there exists $n$ and $i$ such that $\left(\phi^{n}(\alpha), \phi^{n}(b)\right)=\left(c_{i}, d_{i}\right)$. Then there exists $G$ a normal representative of $\mathscr{b}$ such that

$$
J(G) \subset\left\{\left(c_{1}, d_{1}\right), \cdots,\left(c_{p}, d_{p}\right)\right\} .
$$

Proof. First suppose that for each $\left(a_{i}, b_{i}\right)$ the integer $n(i)$ such that $\left(\phi^{n(i)}\left(a_{i}\right), \phi^{n(i)}\left(b_{i}\right)\right)=\left(c_{j}, d_{j}\right)$ for some $j$ is positive. Here we can apply the construction of $G$ preceding Lemma 6.3 to $\mathscr{A}^{\prime}=\left\{\left(\phi^{k}\left(a_{i}\right), \phi^{k}\left(b_{i}\right)\right)\right.$ : $0 \leqq k \leqq n(i)\} \cup\left\{\left(\dot{\phi}^{-1}\left(a_{1}\right), \dot{\phi}^{-1}\left(b_{1}\right)\right)\right\} \supset J(F)$, and if we start numbering $\mathscr{A}^{\prime}$ with $\left(c_{1}, d_{1}\right)$ we get $J(G) \subset\left\{\left(c_{1}, d_{1}\right), \cdots,\left(c_{p}, d_{p}\right)\right\}$. In the general case we first construct an $F^{\prime}$ cohomologous to $F$ to which the above argu- 
ment applies. We obtain $F^{\prime}$ by applying our initial argument to $F\left(\phi^{-1}(x)\right)^{-1}$ which determines $\phi_{F}^{-1}$.

COROLLARY 6.6. If there exist complementary intervals $\left(a_{1}, b_{1}\right), \cdots$, $\left(a_{p}, b_{p}\right)$ such that every complementary interval $(a, b)$ has the form $\left(\phi^{k}\left(a_{i}\right), \phi^{k}\left(b_{i}\right)\right)$ for some $i$ and $k$, then there are only a finite number of minimal cohomology class of $(C, \phi)$ for $S_{n}$.

Proof. For every minimal cohomology class $\mathscr{C}$ there exists $F \in$ $\mathscr{C}$ such that $J(F) \subset\left\{\left(a_{1}, b_{1}\right), \cdots,\left(a_{p}, b_{p}\right)\right\}$.

The problem of computing the number of minimal cohomology classes for a given $p$ and $n$ will be the topic of a future paper with William N. Anderson, Jr.

The indexing of $J(F)$ is only crucial when there is an exceptional jump; i.e., there exists a complementary $(a, b)$ such that $F(a) \neq F(b)$ and $F\left(\phi^{-1}(a)\right) \neq F\left(\phi^{-1}(b)\right)$. In this case we always want $(a, b)=\left(a_{1}, b_{1}\right)$. When this is not the case we change the indexing freely, but for convenience we always index counterclockwise. We will write $J(F) \sim$ $J(G)$ provided $J(F)$ and $J(G)$ consist of the same complementary intervals; and if both $F$ and $G$ have an exceptional jump, it occurs at the same complementary interval for both of them. We will say $F$ and $G$ are conjugate if there exists $\sigma \in S_{n}$ such that $F(x)=\sigma^{-1} G(x) \sigma$ for all $x$ in $C$. The second advantage of normal functions in the following:

THeOREM 6.7. Let $F$ and $G$ be normal representatives of minimal cohomology classes. If $J(F) \sim J(G)$, then $F$ and $G$ are cohomologous if and only if $F$ and $G$ are conjugate.

Proof. Let $\alpha: C \rightarrow S_{n}$ continuously such that $G(x)=\alpha(\phi(x))^{-1} F(x) \alpha(x)$ and let $(a, b)$ be a complementary interval such that $\alpha(a) \neq \alpha(b)$ and $(a, b) \neq\left(a_{1}, b_{1}\right)$ where $J(F)=\left\{\left(a_{1}, b_{1}\right), \cdots,\left(a_{q}, b_{q}\right)\right\}$. Then $F\left(\phi^{n}(a)\right)=$ $F\left(\phi^{n}(b)\right)$ for either all $n \geqq 0$ or all $n<0$. In the first case it follows that $\alpha(\phi(a)) \neq \alpha(\phi(b))$ and hence by induction $\alpha\left(\phi^{n}(a)\right) \neq \alpha\left(\phi^{n}(b)\right)$ for all $n \geqq 0$ which contradicts the continuity of $\alpha$. In the second case we obtain the contradiction by using $G\left(\phi^{-1}(x)\right)=\alpha(x)^{-1} F\left(\phi^{-1}(x)\right) \alpha\left(\phi^{-1}(x)\right)$. Thus $\alpha(a)=\alpha(b)$ unless $(a, b)=\left(a_{1}, b_{1}\right)$. Since $\alpha$ is finitely valued, $\alpha$ is not constant if and only if there exist two complementary intervals with $\alpha(a) \neq \alpha(b)$. Therefore, $\alpha$ is constant and $F$ and $G$ are conjugate.

Let $F$ be a normal representative of a minimal cohomology class. The permutations $A_{+}(a, b)$ and $A_{-}(a, b)$ are computable. Let $J(F)=$ $\left\{\left(a_{1}, b_{1}\right), \cdots,\left(a_{p}, b_{p}\right)\right\}$, and let $(a, b)$ be a complementary interval. If $\left(\phi^{n}(a), \phi^{n}(b)\right) \notin J(F)$ for all $n$, then clearly $A_{+}(a, b)=e$ and $A_{-}(a, b)=$ 
e. If $\left(\phi^{n}(a), \phi^{n}(b)\right)=\left(a_{i}, b_{i}\right)$ where $n \neq 0$, then Lemma 2.1 (c) can be applied and we get

$$
A_{+}(a, b)=F\left(b_{i},-n\right) A_{+}\left(a_{i}, b_{i}\right) F\left(a_{i},-n\right)^{-1}
$$

and

$$
A_{-}(a, b)=F\left(b_{i},-n\right) A_{-}\left(a_{i}, b_{i}\right) F\left(a_{i},-n\right)^{-1} .
$$

Now consider $\left(a_{i}, b_{i}\right) \in J(F)$. Then $A_{+}\left(a_{i}, b_{i}\right)=F\left(b_{i}\right)^{-1} F\left(a_{i}\right)$ and $A_{-}\left(a_{i}, b_{i}\right)=$ $e$ for $i>1$. If there is no exceptional jump, then $A_{-}\left(a_{1}, b_{1}\right)=e$. When there is an exceptional jump

$$
A_{-}\left(a_{1}, b_{1}\right)=F\left(\phi^{-1}\left(b_{1}\right)\right) F\left(\phi^{-1}\left(a_{1}\right)\right)^{-1}
$$

because $A_{-}\left(\phi^{-1}\left(a_{1}\right), \phi^{-1}\left(b_{1}\right)\right)=e$. It follows that for $i>1,\left(a_{i}, j\right)$ and $\left(b_{i}, j\right)$ are doubly asymptotic if and only if $A_{+}\left(a_{i}, b_{i}\right) j=j$. But this is certainly not ture for all $j$, and for some $j$ the proximal cell of $\left(a_{i}, j\right)$ contains two points which are not proximal. Thus if $J\left(F^{\prime}\right)$ contains more than one complementary interval, $\left(C, \phi_{F}\right)$ is not locally almost periodic. When $F$ does not have an exceptional jump the same comments can be made about $\left(a_{1}, j\right)$. If there is an exceptional jump, then $\left(\phi^{-1}\left(a_{1}\right), j\right)$ and $\left(\phi^{-1}\left(b_{1}\right), j\right)$ are boubly asymptotic if and only if $F\left(\phi^{-1}\left(b_{1}\right)\right)^{-1} F\left(b_{1}\right)^{-1} F\left(a_{1}\right) F\left(\phi^{-1}\left(a_{1}\right)\right) j=j$. We will say $\left(C_{n}, \phi_{F}\right)$ is trivial if there exists a circular cascade $\left(C^{\prime}, \phi^{\prime}\right)$ and $r>0$ such that $\left(C_{n}, \phi_{F}\right)$ is isomorphic to $\left(C_{r}^{\prime}, \phi_{G}^{\prime}\right)$ where $G(x)=(1,2, \cdots, r)$ for all $x$.

THEOREM 6.8. Let $F$ be a normal representative of a minimal cohomology class. Then $\left(C_{n}, \phi_{F}\right)$ is trivial if and only if $|J(F)|=1$ and $F(x) F(y)=F(y) F(x)$ for all $x$ and $y$ in $C$.

Proof. First suppose $\left(C_{n}, \phi_{F}\right)$ is trivial. It follows that it is locally almost periodic and $\left(\phi^{-1}\left(a_{i}\right), j\right)$ and $\left(\phi^{-1}\left(b_{i}\right), j\right)$ are doubly asymptotic for all $j$. Consequently $|J(F)|=1$ and $F\left(\phi^{-1}\left(b_{1}\right)\right)^{-1} F\left(b_{1}\right)^{-1} F\left(a_{1}\right) F\left(\phi^{-1}\left(a_{1}\right)\right)=1$. Since $|J(F)|=1, F(x)$ takes on only two values, $F\left(b_{1}\right)=F\left(\phi^{-1}\left(a_{1}\right)\right)$, and $F\left(a_{1}\right)=F\left(\phi^{-1}\left(b_{1}\right)\right)$.

Now suppose $\left|J\left(F^{\prime}\right)\right|=1$ and $F(x) F(y)=F(y) F(x)$ for all $x$ and $y$. Hence $(a, j)$ and $(b, j)$ are doubly asymptotic where $(a, b)$ is any complementary interval except $\left(a_{1}, b_{1}\right)$ which is the only complementary interval in $J(F)$. It follows that $P^{\prime} \subset \Delta$. If we can find $N$ such that the minimal sets of $\left(C_{n}, \phi_{F}^{N}\right)$ are circular cascade, we will be done. Let $N=c\left(\phi_{F}\right)$. We can apply Theorem 4.1 [5] to finish the proof provided $\left(C_{n}, \phi_{F}\right)$ and $\left(C_{n}, \phi_{F}^{N}\right)$ have the same equicontinuous structure relation. Because $\left(C_{n}, \phi_{F}\right)$ is minimal we know that its equicontinuous structure relation coincides with its regionally proximal relation i.e., $E\left(\dot{\phi}_{F}\right)=Q\left(\dot{\phi}_{F}\right)$ [6, Theorem 1.1 and Theorem 1.2 or 4, Theorem 2.12]. 
Since we know that $Q\left(\dot{\phi}_{F}\right)=Q\left(\phi_{F}^{N}\right) \subset E\left(\phi_{F}^{N}\right) \subset E\left(\dot{\phi}_{F}\right)$, it follows that $E\left(\dot{\phi}_{F}\right)=E\left(\dot{\phi}_{F}^{N}\right)$.

\section{REFERENCES}

1. J. Auslander, Endomorphisms of minimal sets, Duke Math. J., 30 (1963), 605-614.

2. N. Bourbaki, General Topology, Herman, Paris, and Addison-Wesley, Reading, Mass., 1966.

3. I. U. Bronstein, Extensions of transformation groups, Sibirskii Mathematiceskii Zurnal, 9 (1968), 13-20 (Russian); Siberian Mathematical Journal, 9 (1968), 9-14 (English translation).

4. R. Ellis and H. Keynes, A characterization of equicontinuous structure relation, Trans. Amer. Math. Soc., (to appear).

5. N. Markley, Homeomorphisms of the circle without periodic points, Proc. London Math. Soc., 20 (3), (1970), 688-698.

6. W. Veech, The equicontinuous structure relation for minimal abelian transformation groups, Amer. J. Math., 90 (1968), 723-732.

7. - Strict ergodicity in zero dimensional dynamical systems and the KroneckerWeyl theorem mod 2, Trans. Amer. Math. Soc., 140 (1969), 1-33.

8. - Point distal flows, Amer. J. Math., 92 (1970), 205-242.

Received January 28, '1972 and in revised form June 13, 1972. The author was supported by the National Science Foundation GP-12038.

UNIVERSITY OF MARYLAND 



\section{PACIFIC JOURNAL OF MATHEMATICS}

\section{EDITORS}

D. Gilbarg and J. Milgram

Stanford University

Stanford, California 94305

R. A. Beaumont

University of Washington

Seattle, Washington 98105
J. DUGUNDJI* Department of Mathematics

University of Southern California Los Angeles, California 90007

RICHARD ARENS

University of California

Los Angeles, California 90024

\section{ASSOCIATE EDITORS}

E. F. BeCKenbach

B. H. NeUMaNN

F. WOLF

K. Yoshida

\section{SUPPORTING INSTITUTIONS}

UNIVERSITY OF BRITISH COLUMBIA

UNIVERSITY OF SOUTHERN CALIFORNIA

CALIFORNIA INSTITUTE OF TECHNOLOGY

UNIVERSITY OF CALIFORNIA

MONTANA STATE UNIVERSITY

STANFORD UNIVERSITY

UNIVERSITY OF TOKYO

UNIVERSITY OF NEVADA

UNIVERSITY OF UTAH

NEW MEXICO STATE UNIVERSITY

WASHINGTON STATE UNIVERSITY

OREGON STATE UNIVERSITY

UNIVERSITY OF OREGON

OSAKA UNIVERSITY

UNIVERSITY OF WASHINGTON

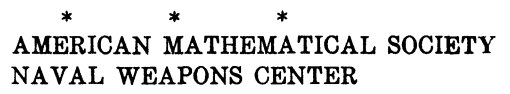

* C. DePrima will replace J. Dugundji until August 1974. 


\section{Pacific Journal of Mathematics}

\section{Vol. 47, No. $1 \quad$ January, 1973}

K. Adachi, Masuo Suzuki and M. Yoshida, Continuation of holomorphic

mappings, with values in a complex Lie group ....................

Michael Aschbacher, A characterization of the unitary and symplectic groups

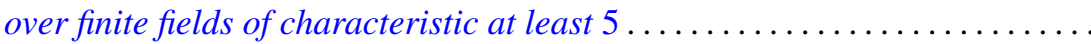

Larry Eugene Bobisud and James Calvert, Energy bounds and virial theorems for abstract wave equations....................................

Christer Borell, A note on an inequality for rearrangements ................

Peter Southcott Bullen and S. N. Mukhopadhyay, Peano derivatives and general

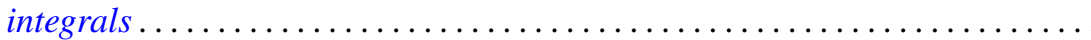

Wendell Dan Curtis, Yu-Lee Lee and Forrest Miller, A class of infinite dimensional subgroups of $\operatorname{Diff}^{r}(X)$ which are Banach Lie groups .........

Paul C. Eklof, The structure of ultraproducts of abelian groups ...............

William Alan Feldman, Axioms of countability and the algebra $C(X) \ldots \ldots \ldots$

Jack Tilden Goodykoontz, Jr., Aposyndetic properties of hyperspaces...........

George Grätzer and J. Płonka, On the number of polynomials of an idempotent algebra. II ...........................................

Alan Trinler Huckleberry, The weak envelope of holomorphy for algebras of

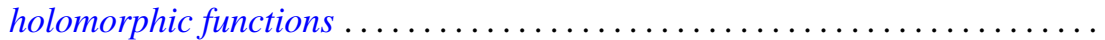

John Joseph Hutchinson and Julius Martin Zelmanowitz, Subdirect sum decompositions of endomorphism rings . . . . . . . . . . . . . . . .

Gary Douglas Jones, An asymptotic property of solutions of

$y^{\prime \prime \prime}+p y^{\prime}+q y=0$.

Howard E. Lacey, On the classification of Lindenstrauss spaces .

Charles Dwight Lahr, Approximate identities for convolution measure algebras.

George William Luna, Subdifferentials of convex functions on Banach

spaces.

Nelson Groh Markley, Locally circular minimal sets. .

Robert Wilmer Miller, Endomorphism rings of finitely generated projective modules

Donald Steven Passman, On the semisimplicity of group rings of linear

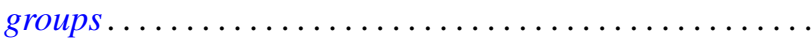

Bennie Jake Pearson, Dendritic compactifications of certain dendritic spaces.

Ryōtarō Satō, Abel-ergodic theorems for subsequences ...... .

Henry S. Sharp, Jr., Locally complete graphs. . .

Harris Samuel Shultz, A very weak topology for the Mikusinski field of

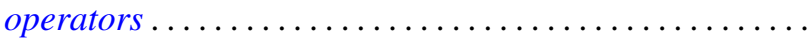

Elena Stroescu, Isometric dilations of contractions on Banach spaces ...

Charles W. Trigg, Versum sequences in the binary system ... . .

William L. Voxman, On the countable union of cellular decompositions of n-manifolds 